\title{
Colgajo DIEP de cobertura tras mastectomía de limpieza paliativa en cáncer de mama localmente avanzado
}

\author{
DIEP flap reconstruction after palliative curettage mastectomy \\ in locally advanced breast cancer
}

\author{
J. Arredondo ${ }^{1}$, N. Rodríguez-Spiteri ${ }^{2}$, W. Torre ${ }^{3}$, C. Aubá ${ }^{4}$, N. Pedano ${ }^{1}$, F.M. Regueira ${ }^{2}$
}

\section{RESUMEN}

Fundamento. La mastectomía de limpieza está indicada con carácter paliativo en el cáncer de mama localmente avanzado. Para cerrar el defecto cutáneo puede ser necesario el empleo de un injerto. Mostramos nuestra experiencia con el uso de un colgajo DIEP (Deep Inferior Epigastric artery Perforators) de cobertura tras la realización de una mastectomía de gran extensión.

Material y métodos. Se muestra el caso de una paciente con un tumor de mama muy avanzado localmente, que era subsidiaria de un tratamiento quirúrgico agresivo tras la administración de quimioterapia.

Resultados. Se consideró que el cierre que puede ofrecer un colgajo realizado con el músculo dorsal ancho esta insuficiente, por lo que se realizó un DIEP. La paciente presentó una evolución postoperatoria satisfactoria.

Conclusiones. El colgajo DIEP de cobertura ofrece una gran extensión cutánea. Puede ser un recurso en casos muy seleccionados de tumores de mama localmente avanzados, en los que la cirugía se convierte en la principal arma terapéutica.

Palabras clave. Mastectomía de limpieza. Mastectomía de salvamento. Cáncer de mama localmente avanzado. DIEP de cobertura.

\begin{abstract}
Background. Curettage mastectomy is indicated as a palliative treatment in locally advanced breast cancer. We present our experience with the use of a DIEP flap reconstruction following extensive mastectomy.

Methods. We report the case of a patient with very advanced local breast cancer that was subsidiary to aggressive palliative surgery after chemotherapy.

Results. It was considered that the closure that could be performed with the latissimus dorsi muscle was unsuitable, therefore a DIEP flap was performed. The patient was discharged uneventfully.

Conclusion. The DIEP reconstruction offers great cutaneous extension. It can be a resource in highly selected cases of locally advanced breast cancer in which surgery becomes the main treatment.
\end{abstract}

Key words. Curettage mastectomy. Salvage mastectomy. Locally advanced breast cancer. DIEP.
1. Cirugía General. Clínica Universidad de Navarra.

2. Área de Patología Mamaria. Clínica Universidad de Navarra.

3. Cirugía Torácica. Clínica Universidad de Navarra.

4. Cirugía Plástica. Clínica Universidad de Navarra.

Recepción: 2 de noviembre de 2012

Aceptación provisional: 26 de noviembre de 2012

Aceptación definitiva: 5 de diciembre de 2012

\author{
Correspondencia: \\ Jorge Arredondo \\ Departamento de Cirugía General \\ Clínica Universidad de Navarra \\ Avda. Pío XII, 36 \\ 31008 Pamplona \\ E-mail: jarredon@unav.es
}

\section{Conflicto de intereses.}

Los autores declaran no tener conflicto de intereses. 


\section{INTRODUCCIÓN}

La mastectomía de limpieza, indicada con carácter paliativo en el cáncer de mama localmente avanzado, plantea un importante problema quirúrgico: el cierre de su defecto una vez realizada una extirpación de gran magnitud. En estos casos después de la mastectomía de limpieza en que no es posible un cierre inmediato, puede ser necesario el empleo de un injerto para conseguir el cierre cutáneo. El injerto más frecuentemente empleado es el del músculo dorsal ancho ${ }^{1-3}$, recobrando especial importancia a partir de la década de los $70^{4,5}$. Sin embargo en ocasiones el defecto post-resección es de una magnitud tal que este injerto no es suficiente. En este trabajo se presenta la experiencia con el empleo de colgajos dermograsos abdominales libres microvascularizados tipo DIEP (colgajo microquirúrgico de perforantes de la arteria epigástrica inferior profunda) como coberura tras una mastectomía de limpieza de gran extensión.

\section{CASO CLÍNICO}

Se presenta el caso clínico de una paciente con cáncer de mama localmente muy avanzado que era subsidiaria de un tratamiento quirúrgico paliativo agresivo. Se planificó una extensa resección para extirpar toda la enfermedad visible, que precisaba una cobertura con un colgajo especialmente amplio. Se consideró que el cierre que se podía realizar con el músculo dorsal ancho no era el adecuado, motivo por el que se empleó un colgajo tipo DIEP.

Se trata de una mujer de 49 años con carcinoma inflamatorio de mama izquierda estadio IIIB (T4 N1 M0). Al diagnóstico presentaba un tumor exofítico, ulcerado y necrótico, palpándose adenopatías axilares pétreas (Fig. 1a). Recibió tratamiento con quimioterapia neoadyuvante con 4 ciclos de epirrubicina y ciclofosfamida seguido de 4 ciclos de Docetaxel y Capecitabina. Dada la respuesta parcial se inició radioterapia preoperatoria, alcanzando una dosis total de 64 Gy, administrándose de manera concomintante Paclitaxel y Carboplatino semanal. Con los tratamientos de quimioterapia y radioterapia se observó disminución de la masa torácica, presentando una lesión ulcerada en hemitórax izquierdo de $7 \mathrm{~cm}$ (Fig. 1b) con la que se planteó la intervención tras haber realizado pruebas de estadiaje, que descartaron enfermedad a distancia.

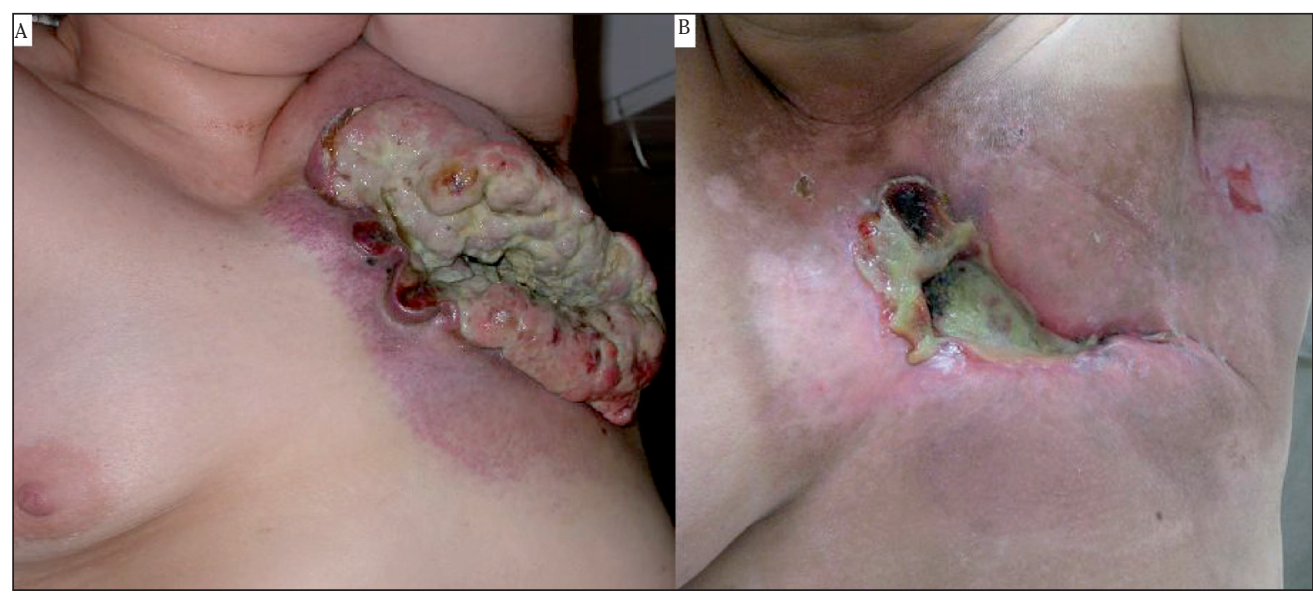

Figura 1: A. Tumor exofítico, ulcerado y necrótico antes del tratamiento neoadyuvante; B. Tumor tras la quimio-radioterapia, mostrando una lesión ulcerada.

Fue necesaria una mastectomía de limpieza con una resección muy amplia para conseguir extirpar toda la enfermedad tumoral macroscópica. La paciente fue intervenida por un equipo multidisciplinar integrado por cirujanos generales, plásticos y torácicos. La cobertura del amplio defecto creado post-mastectomía después de la resección se realizó mediante un colgajo 
DIEP. El objetivo de este colgajo DIEP no fue la reconstrucción mamaria, sino exclusivamente la cobertura cutánea.

Se realizó mastectomía y resección de pared torácica incluyendo tercio medio e inferior esternal y arcos costales derechos e izquierdos por afectación macroscópica de los mismos. Así mismo se realizó linfadenectomía axilar de los niveles 1 y 3 . El defecto torácico post-resección tenía $15 \mathrm{~cm}$ de diámetro con comunicación de ambas pleuras. La reconstrucción de la pared torácica se hizo con una malla de Prolene cementada (Fig. 2). El defecto cutáneo medía 25 x $20 \mathrm{~cm}$ y para su cobertura cutánea se realizó un colgajo DIEP vascularizado por la arteria mamaria derecha sobre la pared reconstruida. La intervención duró 14 horas y 14 minutos. La pieza pesó $960 \mathrm{~g}$. El diagnóstico final fue de carcinoma ductal in- filtrante de mama poco diferenciado residual post-tratamiento (grado de respuesta de Miller III), ER/PR positivo, HER-2 negativo, con afectación del margen de resección de segunda costilla izquierda y metástasis en el ganglio linfático de la mamaria interna (grado de respuesta ganglionar C). El colgajo DIEP no presentó alteraciones de la coloración ni de la perfusión, retirándose los drenajes el sexto día tras la intervención. La zona donante no presentó complicaciones. El postoperatorio transcurrió sin incidencias, lo que permitió que la paciente fuera dada de alta a los 15 días. En la Figura 3 se observa el estado del colgajo DIEP al finalizar la intervención (Fig. 3a) y en la primera revisión postoperatoria (Fig. 3b). Con un seguimiento de 44 meses la paciente está viva y sin signos de recidiva y mantiene tratamiento con Letrozol.

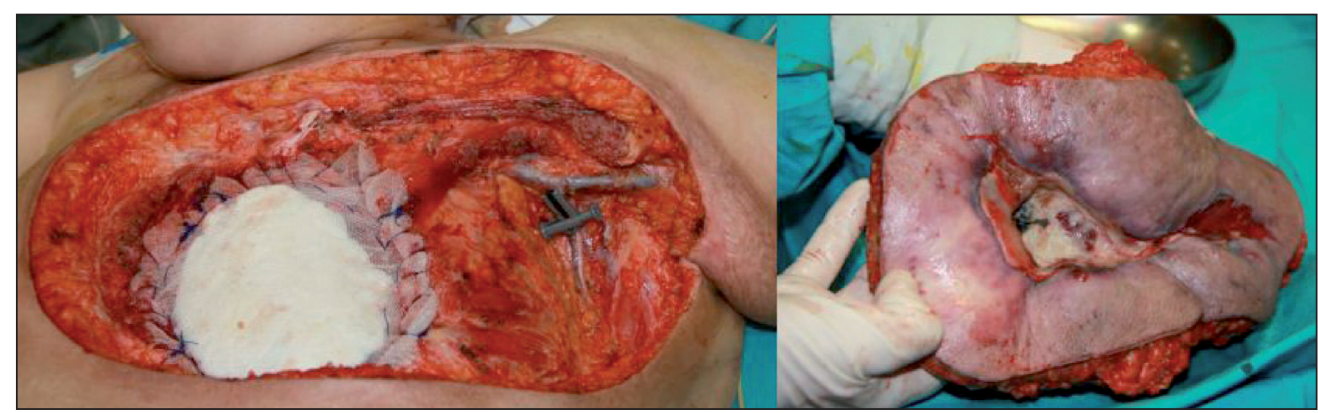

Figura 2. Reconstrucción de pared y espécimen resecado.

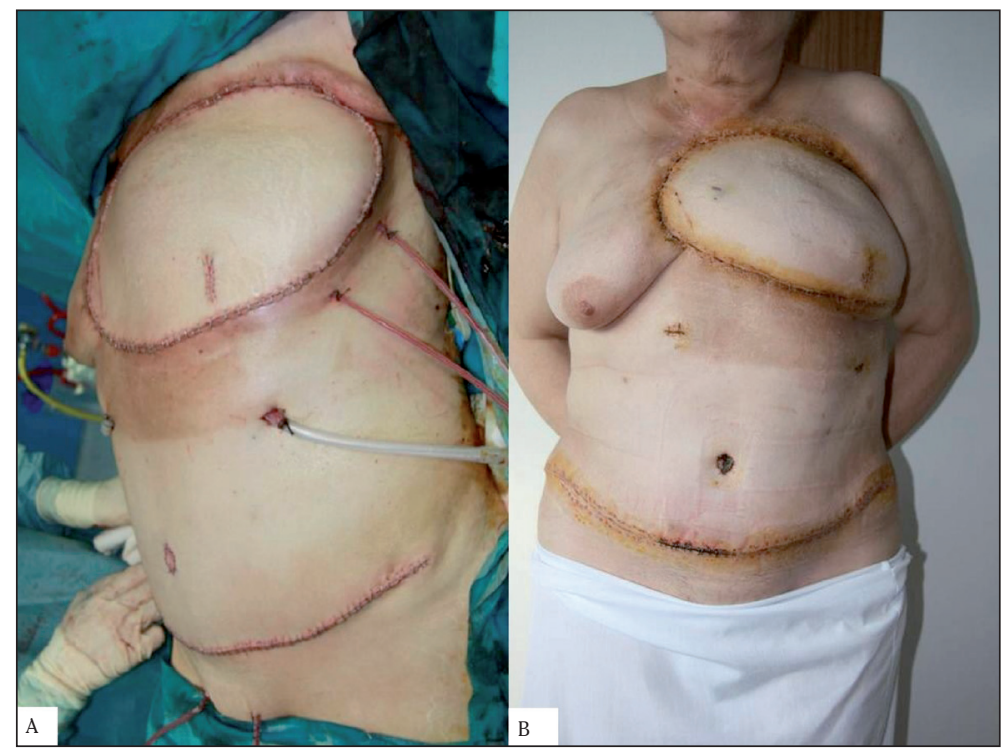

Figura 3: A. Estado del colgajo DIEP al finalizar la intervención; B. Estado del colgajo DIEP en la primera revisión postoperatoria. 


\section{DISCUSIÓN}

La mastectomía de limpieza es una intervención que se empleó muy frecuentemente en el pasado y que consistía en la extirpación de toda la mama, los ganglios axilares, el músculo pectoral y, en ocasiones, la pared costal o incluso el brazo. Esta intervención en la actualidad está casi desterrada debido al diagnóstico precoz del cáncer de mama que hace los tumores localmente avanzados se presenten en porcentajes muy bajos y también a que los tumores diagnosticados en este estadio se tratan, habitualmente con buena respuesta, con quimioterapia y, en ocasiones, radioterapia. Sin embargo, en algunos pacientes seleccionados sigue siendo necesaria una mastectomía de limpieza. Se trata de pacientes en los que no se consigue una buena respuesta a la quimioterapia o en aquellos en los que la enfermedad residual después de un tratamiento neoadyuvante es muy extensa. En todos estos pacientes con carcinoma de mama localmente avanzado el objetivo de la cirugía es la extirpación de toda la enfermedad tumoral visible. Por tanto, puede ser necesaria una extirpación de grandes dimensiones en las que el cierre primario de la herida sea prácticamente imposible. En muchos casos de amplia extirpación cutánea la indicación habitual es recurrir al colgajo del dorsal ancho para su cobertura. Sin embargo, cuando la extirpación es muy extensa el colgajo de dorsal ancho puede ser insuficiente, ya que con él se puede obtener una isla cutánea de aproximadamente $8 \times 20 \mathrm{~cm}$, destacando como efecto secundario el riesgo de isquemia de la piel de la espalda y la asimetría dorsal ${ }^{6}$. En la paciente el defecto cutáneo era mucho mayor y se consideró que el colgajo del dorsal no era suficiente. Por dicho motivo se pensó en realizar un colgajo DIEP, dado que ofrece una isla cutánea de aproximadamente $24 \times 14 \mathrm{~cm}^{7}$, por lo que permite que la resección se adecúe a los requerimientos oncológicos, cuando el área es amplia, como en el caso que nos ocupa.

Para la realización de un colgajo DIEP se precisa llevar a cabo una intervención compleja desde el punto de vista técnico y que supone un mayor tiempo quirúrgico. En nuestro centro se habían realizado más de 100 casos con escasa tasa de complicaciones antes de emplearlo en la primera paciente de este trabajo.

El colgajo DIEP ofrece una serie de ventajas, como son la reconstrucción completamente autógena, la preservación del músculo recto anterior y de la fascia muscular -con la minimización del riesgo herniario-, simetría abdominal, escaso dolor postoperatorio y una mayor longitud del pedículo vascular, otorgando cierta flexibilidad en el posicionamiento del colgajo en la zona receptora ${ }^{8-12}$.

\section{BIBLIOGRAFÍA}

1. WiCKMAN M. Breast reconstruction-past achievements, current status and future goals. Scand J Plast Reconstr Surg Hand Surg 1995; 29: 81-100.

2. URoskie TW, ColEn LB. History of breast reconstruction. Semin Plast Surg 2004; 18: 65-69.

3. TANSINI I. Sopra il moi nuovo processo di amputazione Della mamella (in Italian). Gazetta Medica Italiana 1906; 57: 141

4. EsCUDERO FJ. Historical evolution of breast reconstruction. An Sist Sanit Navar 2005; 28: $7-18$.

5. Olivari N. The latissimus flap. Br J Plast Surg 1976; 29: 126-128.

6. Breast reconstruction with an autologous latissimus flap. Spear. En: Surgery of the breast. Chapter 40. Philadelphia. 2006.

7. GAGNON AR, BlondeEL PN. Deep and superficial inferior epigastric artery perforator flaps. Cir. Plást. Iberlatinamer 2006; 32: 243-258.

8. Blondeel N, Vanderstraeten GG, Monstrey SJ, Van Landuyt K, Tonnard P, Lysens $R$ et al. The donor site morbidity of free DIEP flaps and free TRAM flaps for breast reconstruction. Br J Plast Surg 1997; 50: 322-330.

9. Nahabedian MY, Momen B, Galdino G, Manson PN. Breast Reconstruction with the free TRAM or DIEP flap: patient selection, choice of flap, and outcome. Plast Reconstr Surg 2002; 110: 466-475.

10. Simon AM, Bouwense CL, McMillan S, Lamb S, HAMmOND DC. Comparison of unipedicled and bipedicled TRAM flap breast reconstructions: assessment of physical function and patient satisfaction. Plast Reconstr Surg 2004; 113: 136-140.

11. Serletti JM, Moran SL. Microvascular reconstruction of the breast. Semin Surg Oncol. 2000; 19: 264-271.

12. Perforator flaps in breast reconstruction. Spear. En: Surgery of the breast. Chapter 55. Philadelphia. 2006. 